\title{
Serologic Evidence of Leptospirosis in Patients Attending a Tertiary Care Hospital, Central Kerala, India- A Retrospective Study
}

\author{
Shoba Kurian Pulikottil ${ }^{1}$, Shanimole Puthenpurayil Ebrahimkutty², Ansu Cijo Alex ${ }^{3}$ \\ ${ }^{1}$ Department of Microbiology, Government Medical College, Kottayam, Kerala, India. \\ 2Department of Microbiology, Government Medical College, Kottayam, Kerala, India. \\ ${ }^{3}$ Department of Microbiology, Government Medical College, Kottayam, Kerala, India.
}

\section{ABSTRACT}

\section{BACKGROUND}

Leptospirosis is a zoonotic disease seen all over the world caused by pathogenic members of the genus Leptospira. Humans are infected by direct or indirect contact with an environment contaminated by urine of infected animals such as rodents, cattle and dogs. As the clinical manifestations of leptospirosis are nonspecific, laboratory diagnostic methods are needed for definitive diagnosis.

\section{METHODS}

This is a retrospective study conducted in the Department of Microbiology, Government Medical College, Kottayam, Kerala, in patients with clinical features of acute febrile illness from January 2015 to December 2018. A total of 2941 blood samples were received for leptospira serology. They were subjected to specific anti leptospira IgM ELISA by Panbio Diagnostics, Brisbane, Australia. In the year 2018, 43 samples received during flood period were subjected to PCR, in addition to antileptospira IgM ELISA. For PCR, blood in EDTA, CSF and urine were sent to Manipal Centre for Virus Research, Karnataka. Twenty-five blood samples were sent for Microscopic Agglutination Test to Department of Veterinary Microbiology, College of Veterinary and Animal sciences, Mannuthy, India. Modified Faine's criteria were assessed based on clinical, epidemiological and laboratory findings.

\section{RESULTS}

Among 2941 cases of acute febrile illness, 777 (26.4\%) cases were serologically positive (IgM ELISA) for leptospirosis. Among 777 cases, maximum cases 481 (61.9 $\%)$ were seen during the year 2018, followed by $106(13.64 \%)$ cases during the year 2016, 104 cases (13.38\%) during the year 2015 and 86 (11.06\%) cases during the year 2017. Maximum cases were seen during the month of August, September, and October. Among 43 cases during flood 2018, 35 were (81.4\%) males and 8 $(18.6 \%)$ were females. Maximum cases were seen in the age group of 41-50 years. Among the 43 cases, fever was present in all the $43(100 \%)$ cases followed by myalgia in $36(83.7 \%)$ cases. Headache was seen in $22(51.16 \%)$ cases, jaundice in $16(37.2 \%)$ cases, cough and breathlessness in 15 (34.9\%) cases, conjunctival suffusion in $13(30.2 \%)$, oliguria in $13(30.2 \%)$ cases, vomiting in $11(25.6 \%)$ cases, abdominal pain in $7(16.3 \%)$ cases, diarrhoea in $4(9.3 \%)$ cases, altered sensorium in $3(6.9 \%)$ cases. Among complications, acute kidney injury was seen in 13 (30.2\%) cases, followed by respiratory injury (ARDS) requiring mechanical ventilation in 10 $(23.3 \%)$ cases and myocarditis in $4(9.3 \%)$ cases.

\section{CONCLUSIONS}

Leptospirosis is a zoonotic disease of protean clinical manifestations with difficulty in diagnosis. Confirmation by diagnostic test is essential so that early diagnosis helps in early treatment and prevention of complications in turn reducing mortality.

\section{KEY WORDS}

Microscopic Agglutination Test, Leptospirosis, Elisa
Corresponding Author: Dr. Shanimole Puthenpurayil Ebrahimkutty, Assistant Professor Department of Microbiology, Government Medical College, Kottayam, Kerala, India. E-mail: shanisdr@gmail.com

DOI: $10.14260 /$ jemds/2020/189

Financial or Other Competing Interests: None.

How to Cite This Article: Pulikottil SK, Ebrahimkutty SP, Alex AC. Serologic evidence of leptospirosis in patients attending a tertiary care hospital, Central Kerala, India- a retrospective study. J. Evolution Med. Dent. Sci. 2020;9(11):879-883, DOI: 10.14260/jemds/2020/189

Submission 11-04-2019, Peer Review 19-02-2020, Acceptance 26-02-2020, Published 16-03-2020.

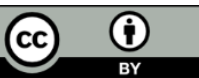




\section{BACKGROUND}

Leptospirosis is a zoonotic disease caused by the spirochete Leptospira. It is a worldwide distributed illness with a higher incidence in tropical countries. The clinical manifestations vary from mild undifferentiated illness to severe multiorgan failure. Natural infection occurs in carrier animals such as rodents, cattle, sheep, goat etc. leading to chronic renal infection and the bacteria are shed in urine.[1] Mode of transmission in humans is direct or indirect contact with infected urine of the carrier animal. Indirect exposure is more common mode of transmission i.e. contact with damp soil or water and wet surfaces contaminated with infected animal's urine. ${ }^{[2]}$ The disease has been reported from various states of India during monsoon months. The disease is endemic in Kerala, Tamilnadu, Gujarat, Andaman, Karnataka, Maharashtra. ${ }^{[3],[4]}$ The important epidemiological risk factors are rainfall and contact with contaminated environment. Leptospirosis can be diagnosed only by lab tests as the clinical features are nonspecific.[4] Leptospirosis has been underreported from India due to lack of appropriate diagnostic facilities.[4] Kerala has reported leptospirosis cases from all districts and the disease is a leading cause of mortality, among the infectious diseases. Timely diagnosis and specific therapy can reduce the severity of illness and in turn mortality.

The aim of this study was to determine the prevalence of leptospirosis among patients attending Government Medical College, Kottayam, Kerala, with clinical features of acute febrile illness during the period January 2015 - December 2018.

\section{METHODS}

The present study is a retrospective study conducted in Department of Microbiology, Government Medical College, Kottayam for a period of four years from January 2015December 2018 in patients with features of acute febrile illness. During this period Microbiology lab received 2941 blood samples for leptospira serology. Blood samples were centrifuged at $3000 \mathrm{rpm}$ for 5 minutes. Serum samples were tested for specific anti - leptospira IgM antibodies using the Panbio IgM ELIZA (Panbio Diagnostics, Brisbane, Australia). The test procedure was performed according to the protocol provided along with the diagnostic kit. The results were interpreted according to manufacturer's instructions, ie; values of $<9$ Panbio ELISA units were considered negative, 911 equivocal, and $>11$ positive. For samples showing equivocal results, another blood sample were drawn after a period of 10 days and the test was repeated. Negative and positive samples were kept with each test run. Forty-three samples received during the flood 2018 were subjected to PCR in addition to specific antileptospira IgM antibody. For PCR, $5 \mathrm{ml}$ of blood in EDTA, CSF and urine were collected and sent to Manipal virology institute and research maintaining cold chain. All these samples were evaluated noting their clinical history and modified Faine's criterion was scored and assessed. 25 blood samples were tested for Microscopic Agglutination Test (MAT). Samples were sent to Department of Veterinary Microbiology, College of Veterinary and Animal
Sciences, Mannuthy, India where MAT was carried out following standard procedure using 11 live leptospira reference strains as antigens procured from National Reference Centre, Port Blair.

\section{Statistical Analysis}

Sample size

$n=\frac{4 p q}{d^{2}}$

$\mathrm{p}$ is taken as $24[15], \mathrm{q}=100-\mathrm{p}=76, \mathrm{~d}$ is $20 \%$ of $\mathrm{p}$

$n=\frac{4 \times 24 \times 76}{4.8^{2}}=316$

\section{RESULTS}

During the study period, 2015 to 2018, among 2941 cases of acute febrile illness, 777 (26.4\%) cases were serologically positive (IgM ELISA) for leptospirosis. Among 777 cases, maximum cases 481 (61.9\%) were seen during the year 2018, followed by 106 (13.64 \%) cases during the year 2016, 104 cases (13.38\%) during the year 2015 and 86 (11.06\%) cases during the year 2017 [Table 1]. During the year 2015, among 556 cases tested, 104 (18.7\%) were serologically positive for leptospirosis. During the year 2016, among 605 cases tested, $106(17.5 \%)$ were serologically positive for leptospirosis. During the year 2017, among 611 cases were tested, $86(14.07 \%)$ were serologically positive for leptospirosis. During the year 2018, among 1269 cases tested, 481 (37.9\%) cases were serologically positive for leptospirosis [Table 1].

Maximum cases were seen during the month of August, September, and October. During the year 2018, 43 cases were clinically evaluated. Among 43 cases 35 were (81.4\%) males and $8(18.6 \%)$ were females. Maximum cases were seen in the age group of 41-50 years [Table 2]. Major epidemiologic factors noted in these patients were contact with dirty water after rainy season, mainly during flood and contact with animals. Among the 43 cases, fever was present in all the 43 $(100 \%)$ cases followed by myalgia in 36 (83.7 \%) cases. Headache was seen in $22(51.16 \%)$ cases, jaundice in 16 (37.2\%) cases, cough and breathlessness in 15 (34.9\%) cases, conjunctival suffusion in 13 (30.2\%), oliguria in $13(30.2 \%)$ cases, vomiting in $11(25.6 \%)$ cases, abdominal pain in 7 $(16.3 \%)$ cases, diarrhoea in $4(9.3 \%)$ cases, altered sensorium in 3 (6.9\%) cases [Table 3]

Among complications, acute kidney injury was seen in 13 (30.2\%) cases, followed by respiratory injury (ARDS) requiring mechanical ventilation in 10 (23.3\%) cases, myocarditis in $4(9.3 \%)$ cases [Table 4]. All the 43 cases $(100 \%)$ had a modified Faine's score more than 26 and all the 43 cases $(100 \%)$ could diagnose as presumptive diagnosis of leptospirosis. Among 43 cases, specific IgM antileptospira antibody ELISA was positive in 31 (72.3 \%) cases, PCR alone was in $5(11.6 \%)$ cases, both IgM ELISA \& PCR positive in 7 (16.3\%) cases [Table 5]. Among the PCR alone positive cases, positivity was found in blood samples. Among the 7 PCR\& IgM ELISA positive cases, PCR was found to be positive in four blood samples, two urine samples and in both blood and 
urine of a single case. Microscopic agglutination was done for 25 samples. 18 samples were found to be positive for MAT. By the microscopic agglutination test (MAT), following serovars were identified viz; Icterohaemorrhagiae, Cyanopteri, Australis, Canicola, Pomona, Javanica, Sejroe, Autumnalis, Gryppotyphosa and Hebdomadis.

All the 43 cases $(100 \%)$ had a modified Faine's score more than 26 and all the 43 cases $(100 \%)$ could diagnose as presumptive diagnosis of leptospirosis. Among 43 cases, specific IgM antileptospira antibody ELISA was positive in 31 $(72.3 \%)$ cases, PCR alone was in $5(11.6 \%)$ cases, both IgM ELISA \& PCR positive in 7 (16.3\%) cases. Among 25 samples where MAT was done, 18 (72\%) samples were found to be positive. By the microscopic agglutination test (MAT), following serovars were identified viz; Icterohaemorrhagiae, Cyanopteri, Australis, Canicola, Pomona, Javanica, Sejroe, Autumnalis, Gryppotyphosa and Hebdomadis.

\begin{tabular}{|cccc|}
\hline Year & Number Tested & Positive & Percentage \\
2015 & 556 & 104 & 18.7 \\
2016 & 605 & 106 & 17.5 \\
2017 & 611 & 86 & 14.07 \\
2018 & 1269 & 481 & 37.9 \\
Total & $\mathbf{2 9 4 1}$ & $\mathbf{7 7 7}$ & $\mathbf{2 6 . 4}$ \\
\hline \multicolumn{4}{c}{ Table 1. Serological Positivity-Specific } \\
Antileptospira IgM Antibody Elisa \\
\hline
\end{tabular}

\begin{tabular}{|ccc|}
\hline Age & No. & Percentage \\
$1-10$ & 0 & 0 \\
$11-20$ & 2 & 4.7 \\
$21-30$ & 3 & 6.9 \\
$31-40$ & 5 & 11.7 \\
$41-50$ & 16 & 37.2 \\
$51-60$ & 9 & 20.9 \\
$61-70$ & 6 & 13.9 \\
$71-80$ & 2 & 4.7 \\
Total & $\mathbf{4 3}$ & $\mathbf{1 0 0}$ \\
\hline Table 2. Age Wise Distribution of Leptospirosis
\end{tabular}

\begin{tabular}{|cccc|}
\hline SI. No. & Symptoms & No & Percentage \\
1 & Fever & 43 & 100 \\
2 & Myalgia & 36 & 83.72 \\
3 & Headache & 22 & 51.16 \\
4 & Jaundice & 16 & 37.20 \\
5 & Cough \& breathlessness & 15 & 34.88 \\
6 & Conjunctival suffusion & 13 & 30.23 \\
7 & Oliguria & 13 & 30.2 \\
8 & Vomiting & 11 & 25.58 \\
9 & Abdominal pain & 7 & 16.27 \\
10 & Diarrhoea & 4 & 9.3 \\
11 & Altered sensorium & 3 & 6.97 \\
\hline \multicolumn{4}{|c|}{} \\
\hline \multicolumn{4}{|c|}{ Table 3. Clinical Manifestations of Leptospirosis } \\
\hline
\end{tabular}

\begin{tabular}{|cccc|}
\hline Sl. No. & Complications & No. & \% \\
1 & Renal failure & 13 & 30.2 \\
2 & Respiratory failure requiring mechanical ventilation & 10 & 23.3 \\
3 & Myocarditis & 4 & 9.3 \\
4 & Hepatorenal injury & 3 & 6.9 \\
\hline \multicolumn{3}{|c|}{ Table 4. Complications of Leptospirosis } \\
\hline
\end{tabular}

\begin{tabular}{|cccc|}
\hline Name of Test & Total Samples Tested & Positivity (no) & $\%$ \\
PCR & 43 & 5 & 11.6 \\
IgM ELISA & 43 & 31 & 72.1 \\
PCR \& IgM ELISA & 43 & 7 & 16.3 \\
\hline \multicolumn{4}{|c|}{ Table 5. Comparison of PCR, Anti- Leptospira IgM ELISA } \\
\hline
\end{tabular}

\section{DISCUSSION}

In this study, the serological prevalence of leptospirosis during 2015-2018 is 26.4\%. During the period 2015-2017, there was a sustained rise of leptospirosis prevalence ranging from $14.07 \%$ to $18.7 \%$ with an abrupt rise during the year 2018 ie; $37.9 \%$. In a study conducted in Calicut, Kerala in
2006, the prevalence rate in healthy adult was $24 \% .{ }^{[5]}$ Leptospirosis is considered as an occupational disease of persons engaged in agriculture, sewage waste, forestry and animal slaughtering. The Central Kerala is a midland region with mountain terrains, low lying lands and rivers. The people are engaged in agriculture mainly paddy, live- stock and animal husbandry. Period between June and September is considered as monsoon. Leptospirosis is endemic in many parts of Kerala. In the present study maximum cases were seen during August, September and October ie; during monsoon and post monsoon period. Leptospirosis is endemic throughout the world. Outbreak may follow periods of rainfall. Rainfall, contact with contaminated water are known risk factors of leptospirosis.[6]

In 2018 , the seropositivity was $37.9 \%$ with an abrupt rise during the month of August (20.31\%), September (27.28\%), October (22.8\%). During November 2018 the seropositivity was (19.9\%) and in, December 2018 it was (18.04\%). This was due to the heavy floods during the month of June and August 2018. Kerala gets heavy rains and intermittent floods from April to October. Intermittent flooding of low-lying areas leads to repeated flushing out of the forest and farmlands and the rodent burrows there into all water sources including ponds, streams, rivers and canals where leptospira can survive for months. This contamination of surface water can cause monsoon outbreaks as well as sporadic cases throughout the year. Epidemics of leptospirosis during monsoon months have been reported from Kerala.[7,8] During the heavy flood period in 2018, 43 cases of acute febrile illness were closely evaluated clinically. Among these 43 cases (81.4\%) were males and $18.6 \%$ were females. This may be due to the fact that males were mainly involved in outdoor activities and rescue operations. This finding was in correlation with the study of Kannan et al and WHO.[9,10] Maximum cases were seen in the age group of 4150 years. This finding was in correlation with the study of Kannan et al and Parmar G et al.[11,12]

In the present study the most common symptoms were fever, myalgia and headache, followed by jaundice, cough and breathlessness, conjunctival suffusion and oliguria. Abdominal pain, Vomiting, altered sensorium and bloody diarrhoea were present. Fever was present in all the cases. Similar pattern was observed in the studies of Sethi et al and Charita Thalva et al. Clinical spectrum of disease is variable based on the serotype infected, age and the immunological status of the individual. [5,12] The characteristic pathogenic mechanism in leptospirosis is a systemic vasculitis leading to pulmonary haemorrhage, ischemia of the renal cortex and tubular epithelial necrosis, and destruction of the hepatic architecture, resulting in jaundice and liver cell injury, with or without necrosis. Immune mediated mechanism also influences the severity of symptoms.[6] The most common complication observed in this study was acute kidney injury followed by ARDS requiring mechanical ventilation. Myocarditis and hepatorenal injury were also present. This finding was in correlation with study of Sethi et al in 2010,[5] ThalvaC.et al in 2017.[12]

In the present study, out of 43 cases PCR alone was positive only in (5/43) $11.6 \%$ cases. Here PCR positivity was found in blood samples alone, not in CSF and urine samples. It was also observed that in all the blood PCR positive cases, duration of fever was less than five days. In the present study 
out of 43 cases, specific antileptospira IgM ELISA were positive in $31(72.1 \%)$ cases Here the duration of fever was more than seven days of duration. In the present study., out of 43 cases, both PCR and specific anti leptospira IgM ELISA were positive in 7 (16.3\%) cases i.e. four from blood, two from blood and CSF, one from blood and urine samples. In all these cases duration of fever was 6 - 8 days. In the present study, MAT was done in 25 samples. Only 18 (72\%) samples were positive where duration of clinical symptoms were more than 10 days. From this study, it was also observed that in acute phase of illness the choice of test is PCR. But this test is expensive, not available in peripheral hospitals, not even in all tertiary care hospitals. Moreover, PCR cannot detect the infecting serovar.

Serological demonstration of antibodies is the main mode of diagnosis used in most of the countries. Antibodies become detectable by the $6^{\text {th }}$ to $10^{\text {th }}$ day of disease and generally reach peak within 3 to 4 weeks. Antibodies levels then gradually decrease but may remain detectable for years. Demonstration of IgM antibody by ELISA is the method used. This is a genus specific test that helps in the rapid diagnosis of current infection. It is simple, more sensitive and become positive earlier than MAT.[13] But ELISA does not give an indication of the infecting serovar. MAT is a serovar specific test and is the gold standard test for the diagnosis of leptospirosis because of its unsurpassed diagnostic specificity. But is of low sensitivity in diagnosing current infection. Even if MAT is considered as the gold standard method and reference method for the diagnosis of leptospirosis, it is not helpful in diagnosis during first week of illness because agglutinating antibodies rise and peak only in $2^{\text {nd }}$ or $3^{\text {rd }}$ week. Moreover, MAT is available in referral centers and is a complex test to perform.[13]

Clinical manifestation of Leptospirosis varies from mild undifferentiated illness, a self-limited systemic illness that is seen in approximately $90 \%$ infections, and fatal multi system failure ie; renal failure and liver failure (Weil's disease) and haemorrhagic pneumonitis. In some patients, the disease shows two distinct phases, an initial septicaemic phase lasting for 5-7 days and an immune phase lasting for 4-30 days. However, in many severe cases, the distinction between these two phases is not apparent. During the acute phase, routine laboratory tests are nonspecific, but indicates bacterial infection. During this phase Leptospira can be recovered from blood and cerebrospinal fluid (CSF). Leptospira can also be recovered from urine, beginning about 5-7 days after the onset of symptoms. The disappearance of leptospira from the blood and CSF coincides with the appearance of IgM antibodies. The organism can be detected in almost all tissues and organs and in urine for several weeks depending on the severity of the disease.[6]

The laboratory diagnosis of leptospirosis includes direct detection methods such as dark field microscopy, PCR, culture and indirect methods such as demonstration of antibodies- IgM ELISA, MAT and other rapid tests. For dark field microscopy, blood, urine, and CSF are the useful specimens. The sensitivity and specificity of this method is low ie; $40.2 \%$ and $61.5 \%$ respectively. Microscopy of blood and CSF is of value only during the acute phase of illness. Microscopy of urine is useful only between 10 and 30 days of illness. The draw backs of DGM as a diagnostic tool is that it is difficult to differentiate pathogenic leptospira from saprophytic leptospira. Moreover, experienced hand is required for interpretation. The most definitive way of confirming the diagnosis of leptospirosis is isolation of organism by culture. The specimens used are blood, CSF and urine. Unfortunately, culture will not help in early diagnosis i.e.; during acute phase because primary isolation may be delayed and may take weeks or months to give a positive culture after inoculation into culture medium. Moreover, culture technique is laborious and time consuming

In the present study, Modified Faine's criteria could diagnose all the 43 cases (100\%) as presumptive diagnosis of leptospirosis. This correlates with the findings of Sethi et al from PGI, Chandigarh[5] and Mandal et al.[1] Modified Faine's criteria (2012) is one of the standard criteria for diagnosis of human leptospirosis. In these guidelines, the diagnosis is based on three categories viz. clinical data (part A), epidemiological factors (part B) and bacteriological and lab findings (part $\mathrm{C}$ ). A score of $\mathrm{A}+\mathrm{B}+\mathrm{C}=25$ or more, Part $\mathrm{A}$ or Part $A$ and $B$ score $=26$ or more is a presumptive diagnosis of leptospirosis. A score between 20 and 25 suggests leptospirosis as a possible diagnosis.[4,13,14] As the clinical features of leptospirosis is nonspecific and the diagnostic tests become positive only after one week, this scoring system is valuable in diagnosis of leptospirosis.

\section{CONCLUSIONS}

Human leptospirosis is a disease of protean manifestations. Rain fall and flooding are the most important risk factors. Confirmation by diagnostic tests is essential so that early diagnosis helps in early treatment thereby decreasing mortality and complications. In the first five days of illness PCR is valuable in the diagnosis. In the late phase of acute ilness (5-10 days), rapid tests like IgM ELISA is useful and are sensitive than MAT. Even though MAT is the gold standard for diagnosing leptospirosis, it is useful after 10 days in the early convalescence phase to obtain best results. Since the confirmatory tests like PCR, Culture and MAT is available in higher centres, it is very essential that rapid tests like IgM ELISA are made easily available in both urban and rural health centres for diagnosis of leptospirosis.

\section{REFERENCES}

[1] DebMandal M, Mandal S, Pal NK. Serologic evidence of human leptospirosis in and around Kolkata, India: a clinic epidemiological study. Asian Pacific Journal of Tropical Medicine 2011;4(12):1001-6.

[2] Unnikrishnan D, Pisharady R, Vijayalekshmi N. Prognostic factors in leptospirosis- a study from Kerala, India. Infect Dis Clin Prac 2005;13(3):104-7.

[3] Report of the Brainstorming meeting on Leptospirosis Prevention and control, Mumbai 16-17 February 2006. Joint Publication by office of WHO, Representative to India. New Delhi and Regional Medical Research Centre (ICMR), WHO Collaborating Centre for Diagnosis, Research, Reference and Training on Leptospirosis Portblair, Andaman and Nicobar Islands. 2006. 
[4] Shivakumar S. Indian guidelines for the diagnosis and management of human leptospirosis. Chapter - 7. In: Murugabathan A, edr. API Medicine Update. 23 $3^{\text {rd }}$ edn. 2013: p. 23-9. www.apiindia.org

[5] Sethi S, Sharma N, Kakkar N, et al. Increasing trends of leptospirosis in northern India: a clinic-epidemiological study. PLoS Neglected Tropical Diseases 2010;4(1):e579.

[6] Levett PN, Hakke DA. Leptospira species. Mandell Douglas \& Bennett's Principle and practice of infectious diseases. Vol. 2. $7^{\text {th }}$ edn. Philadelphia: Churchill Livingstone /Elsevier 2010: p. 3059-65.

[7] Pappachan MJ, Sheela M, Aravindan KP. Relation of rainfall pattern and epidemic leptospirosis in the Indian state of Kerala. J Epidemiol Community Health 2004;58(12):1054.

[8] Pappachan MJ, Mathew S, Aravindan KP, et al. Risk factors for mortality in patients with leptospirosis during an epidemic in Northern Kerala. Nat Med J India 2004;17(5):240-2.
[9] Leptospirosis situation in the WHO South -East Asia Region. Communicable Disease Surveillance and response SEA CD -216.

[10] Kannan R, Alex AG. Clinical profile and prognostic factors of leptospirosis: a study of 50 cases from North Kerala, India. Int J Med Sci 2017;5(11):4793-6.

[11] Parmar G, Kava D, Mehta S, et al. Socio-demographic, clinical and laboratory profile of leptospirosis cases registered at Smimer, Surat. Natl J Comm Med 2013;4(3):507-11.

[12] Thalva C, Desmani KK. Socio-demographic, clinical, epidemiological and laboratory profile of cases of leptospirosis at tertiary care hospital: a two year study. International Journal of Community Medicime and Public Health 2017;4(12):4738.

[13] Shivkumar S. Leptospirosis - Current scenario in India. Medicine Update 2008;18:799-809.

[14] Faine S, WHO. Guidelines for the control of Leptospirosis. WHO Offset Publication 1982: p. 67. 